\title{
Third-Order Spectral Characterization of Termite's Emission Track
}

\author{
Juan-José González de-la-Rosa ${ }^{1}$, I. Lloret $^{1}$, Carlos G. Puntonet ${ }^{2}$, \\ A. Moreno ${ }^{1}$, and J.M. Górriz ${ }^{2}$ \\ ${ }^{1}$ University of Cádiz, Electronics Area. \\ Research Group TIC168 - Computational Instrumentation and Industrial Electronics. \\ EPSA. Av. Ramón Puyol S/N. 11202, Algeciras-Cádiz, Spain \\ juanjose.delarosa@uca.es \\ ${ }^{2}$ University of Granada, Department of Architecture and Computers Technology, \\ ESII, C/Periodista Daniel Saucedo. 18071, Granada, Spain \\ carlos@atc.ugr.es
}

\begin{abstract}
A higher-order frequency-domain characterization of termite activity (feeding and excavating) has been performed by means of analyzing diagonal slices of the bi-spectrum. Five sets of signals of different qualities were acquired using a high sensitivity probe-accelerometer. We conclude that it is possible to establish a third-order pattern (spectral track) associated to the termite emissions, and resulting from the impulsive response of the sensor and the body or substratum through which the emitted waves propagate.
\end{abstract}

\section{Introduction}

Termite detection has gained importance in the last decade mainly due to the urgent necessity of avoiding the use of harming termiticides, and to the joint use of new emerging techniques of detection and hormonal treatments, with the aim of performing an early treatment of the infestation. A localized partial infestation can be exterminated after two or three generations of these insects with the aid of hormones [1, 2].

User-friendly equipment is being currently used in targeting subterranean infestations by means of temporal analysis. An acoustic-emission (AE) sensor or an accelerometer is attached to the suspicious structure. Counting the hits produced by the insects and been registered by the accelerometer, the instrument outputs light signals. At the same time, the user can listen to the sounds and perform some pre-processing, like filtering or amplifying. A set of hits is defined as an acoustic event, which in fact constitutes the electronic tracks of the insects.

These instruments are based on the calculation of the root mean square (RMS) value of the vibratory waveform. The use of the RMS value can be justified both by the difficulty of working with raw AE signals in the high-frequency range, and the scarce information about sources and propagation properties of the AE waves. Noisy media and anisotropy makes even harder the implementation 
of new methods of calculation and measurement procedures. A more sophisticated family of instruments make use of spectral analysis and digital filtering [3]. Both have the drawback of the relative high cost and their practical limitations.

In fact, the usefulness of the above prior-art acoustic techniques and equipments depends very much on several biophysical factors. The main one is the amount of distortion and attenuation as the sound travels through the soil $(\sim 600$ $\mathrm{dB} \mathrm{m}^{-1}$, compared with $0.008 \mathrm{~dB} \mathrm{~m}^{-1}$ in the air) 1 . Furthermore, soil and wood are far from being ideal propagation media because of their high anisotropy, nonhomogeneity and frequency dependent attenuation characteristics 3 .

On the other hand, second order statistics and power spectra estimation (the second order spectrum) fail in low SNR conditions even with ad hoc piezoelectric sensors. Spectrum estimation and spectrogram extract time-frequency features, but ignoring phase properties of the signals. Besides, second-order algorithms are very sensitive to noise.

Other prior-art second-order tools, like wavelets and wavelet packets (timedependent technique) concentrate on transients and non-stationary movements, making possible the detection of singularities and sharp transitions, by means of sub-band decomposition. The method has been proved under controlled laboratory conditions, up to a $\mathrm{SNR}=-30 \mathrm{~dB}$ [4].

As an alternative, higher order statistics (HOS), like the bi-spectrum, have proven useful for characterization of termites' emissions, using a synthetics of alarm signals and prior-known symmetrically-distributed noise processes [5], 6]. The conclusions of these works were funded in the advantages of cumulants; in particular, in the capability of enhancing the SNR of a signal buried in symmetrically distributed noise processes. The computational cost paid is the main drawback of the technique. Besides, in the practice the goal is to localize the infestation from weak evidences, in order to prevent greater destruction. For this reason it should be emphasized that non-audible signals have to be detected.

In this paper third-order spectra slices are used to characterize termite emissions. The results help the HOS researcher to better understand the higher-order frequency diagrams; in particular in the field of insect characterization by AE signal processing. The conclusions are based in records which were acquired within the surrounding perimeter of the infestation. The quality of the signals has been established using the criteria of audibility and the levels of quantization used in the digitalizing process by the data acquisition equipment. The accelerometer used is the SP1-L probe from AED2000 instrument, with a high sensitivity and a short band-width.

The paper is structured as follows: Section 2 summarizes the problem of acoustic detection of termites; Section 3 recalls the theoretical background of HOS, focussing on the computational tools. Experiments are drawn in Section 4 which is intended as a tool to interpret results from HOS-based experiments. Finally, conclusions are explained in Section 5. 


\section{Acoustic Detection of Termites}

$\mathrm{AE}$ is defined as the class of phenomena whereby transient elastic waves are generated by the rapid (and spontaneous) release of energy from localized sources within a material, or the transient elastic wave(s) so generated. This energy travels through the material as a stress wave and is typically detected using a piezoelectric transducer, which converts the surface displacement (vibrations) to an electrical signal [4.

Termites use a sophisticated system of vibratory long distance alarm described, among others in, [1. In [4] is is shown one of the impulses within a typical four-impulse burst (alarm signals) and its associated power spectrum of the specie (reticulitermes lucifugus). The carrier frequency of the drumming signal is defined as the main spectral component.

We are concerned about the spectral patterns of the signals; so we do not care about the energy levels. Besides, as a result of the HOS processing, the original energy levels of the signals are lost, but not as the extent of the levels of parasitic noise which are coupled to the signals. Furthermore, the amplitudes of the noise processes, which could be coupled to the waveform under study, are significatively reduced if the probability density function of the stochastic process is symmetrically distributed [5].

Signals from feeding an excavating do not exhibit a concrete time pattern (bursts and impulses equally spaced). They comprise random impulse events that provoke the same response of the sensor and the traversed media. The main handicap is the low intensity of the involved levels. We are concerned about detecting activity by rejecting noise.

In the following we settle the mathematical foundations of HOS for the characterization process.

\section{$3 \quad$ Higher-Order Statistics (HOS)}

The motivation of the poly-spectral analysis is three fold: (a) To suppress Gaussian noise processes of unknown spectral characteristics; the bi-spectrum also suppress noise with symmetrical probability distribution, (b) to reconstruct the magnitude and phase response of systems, and (c) to detect and characterize nonlinearities in time series.

Before cumulants, non-Gaussian processes were treated as if they were Gaussian. Cumulants and their associated Fourier transforms, known as polyspectra [7, reveal information about amplitude and phase, whereas second order statistics (variance, covariance and power spectra) are phase-blind 8 .

The relationship among the cumulants of stochastic signals, $x_{i}$, and their moments can be calculated by using the Leonov-Shiryayev formula. The second-, third-, and fourth-order cumulants are given by [5, [8] equation 1

$$
\begin{aligned}
\operatorname{Cum}\left(x_{1}, x_{2}\right) & =E\left\{x_{1} \cdot x_{2}\right\} . \\
\operatorname{Cum}\left(x_{1}, x_{2}, x_{3}\right) & =E\left\{x_{1} \cdot x_{2} \cdot x_{3}\right\} .
\end{aligned}
$$




$$
\begin{array}{r}
\operatorname{Cum}\left(x_{1}, x_{2}, x_{3}, x_{4}\right)=E\left\{x_{1} \cdot x_{2} \cdot x_{3} \cdot x_{4}\right\} \\
-E\left\{x_{1} \cdot x_{2}\right\} E\left\{x_{3} \cdot x_{4}\right\} \\
-E\left\{x_{1} \cdot x_{3}\right\} E\left\{x_{2} \cdot x_{4}\right\} \\
-E\left\{x_{1} \cdot x_{4}\right\} E\left\{x_{2} \cdot x_{3}\right\} .
\end{array}
$$

In the case of non-zero mean variables $x_{i}$ have to be replaced by $x_{i}-E\left\{x_{i}\right\}$.

Let $\{x(t)\}$ be a $r$ th-order stationary random real-valued process. The $r$ thorder cumulant is defined as the joint $r$ th-order cumulant of the random variables $x(t), x\left(t+\tau_{1}\right), \ldots, x\left(t+\tau_{r-1}\right)$,

$$
\begin{aligned}
& C_{r, x}\left(\tau_{1}, \tau_{2}, \ldots, \tau_{r-1}\right) \\
& \quad=\operatorname{Cum}\left[x(t), x\left(t+\tau_{1}\right), \ldots, x\left(t+\tau_{r-1}\right)\right] .
\end{aligned}
$$

The second-, third- and fourth-order cumulants of zero-mean $x(t)$ can be expressed using equations 1 and 2 , via:

$$
\begin{gathered}
C_{2, x}(\tau)=E\{x(t) \cdot x(t+\tau)\} . \\
C_{3, x}\left(\tau_{1}, \tau_{2}\right)=E\left\{x(t) \cdot x\left(t+\tau_{1}\right) \cdot x\left(t+\tau_{2}\right)\right\} . \\
C_{4, x}\left(\tau_{1}, \tau_{2}, \tau_{3}\right) \\
=E\left\{x(t) \cdot x\left(t+\tau_{1}\right) \cdot x\left(t+\tau_{2}\right) \cdot x\left(t+\tau_{3}\right)\right\} \\
-C_{2, x}\left(\tau_{1}\right) C_{2, x}\left(\tau_{2}-\tau_{3}\right) \\
-C_{2, x}\left(\tau_{2}\right) C_{2, x}\left(\tau_{3}-\tau_{1}\right) \\
-C_{2, x}\left(\tau_{3}\right) C_{2, x}\left(\tau_{1}-\tau_{2}\right) .
\end{gathered}
$$

We assume that the cumulants satisfy the bounding condition given in equation 4

$$
\sum_{\tau_{1}=-\infty}^{\tau_{1}=+\infty} \cdots \sum_{\tau_{r-1}=-\infty}^{\tau_{r-1}=+\infty}\left|C_{r, x}\left(\tau_{1}, \tau_{2}, \ldots, \tau_{r-1}\right)\right|<\infty
$$

The higher-order spectra are usually defined in terms of the $r$ th-order cumulants as their $(r-1)$-dimensional Fourier transforms

$$
\begin{aligned}
S_{r, x} & \left(f_{1}, f_{2}, \ldots, f_{r-1}\right) \\
& =\sum_{\tau_{1}=-\infty}^{\tau_{1}=+\infty} \cdots \sum_{\tau_{r-1}=-\infty}^{\tau_{r-1}=+\infty} C_{r, x}\left(\tau_{1}, \tau_{2}, \ldots, \tau_{r-1}\right) \\
& \cdot \exp \left[-j 2 \pi\left(f_{1} \tau_{1}+f_{2} \tau_{2}+\cdots+f_{r-1} \tau_{r-1}\right)\right] .
\end{aligned}
$$

The special poly-spectra derived from equation 5 are power spectrum $(r=2)$, bi-spectrum $(r=3)$ and try-spectrum $(r=4)$. Only power spectrum is real, the others are complex magnitudes. Poly-spectra are multidimensional functions 
which comprise a lot of information. As a consequence, their computation may be impractical in some cases. To extract useful information one-dimensional slices of cumulant sequences and spectra, and bi-frequency planes are employed in non-Gaussian stationary processes [6].

Once summarized the foundations of the experiment, hereinafter we present que results obtained by means of the tools described here.

\section{Experimental Results}

The data acquisition stage took place in a residential area of the "Costa del Sol" (Málaga-Spain), at the beginning of the reproductive season of the termites. The sensors were attached (plunged) in the soil surrounding affected trees (above the roots). We worked under the hypothesis of having a $500 \mathrm{~m}$-radius of an affected circular perimeter.

The probe SP1-L from the equipment AED2000 has been taken as the reference with a twofold purpose. First, we analyze the power spectra of signals with different qualities, to decide wether it is possible to use second-order spectra for identification purposes. Secondly, we settle down a higher-order detection criterion in the frequency domain which it is supposed to enhance detection.
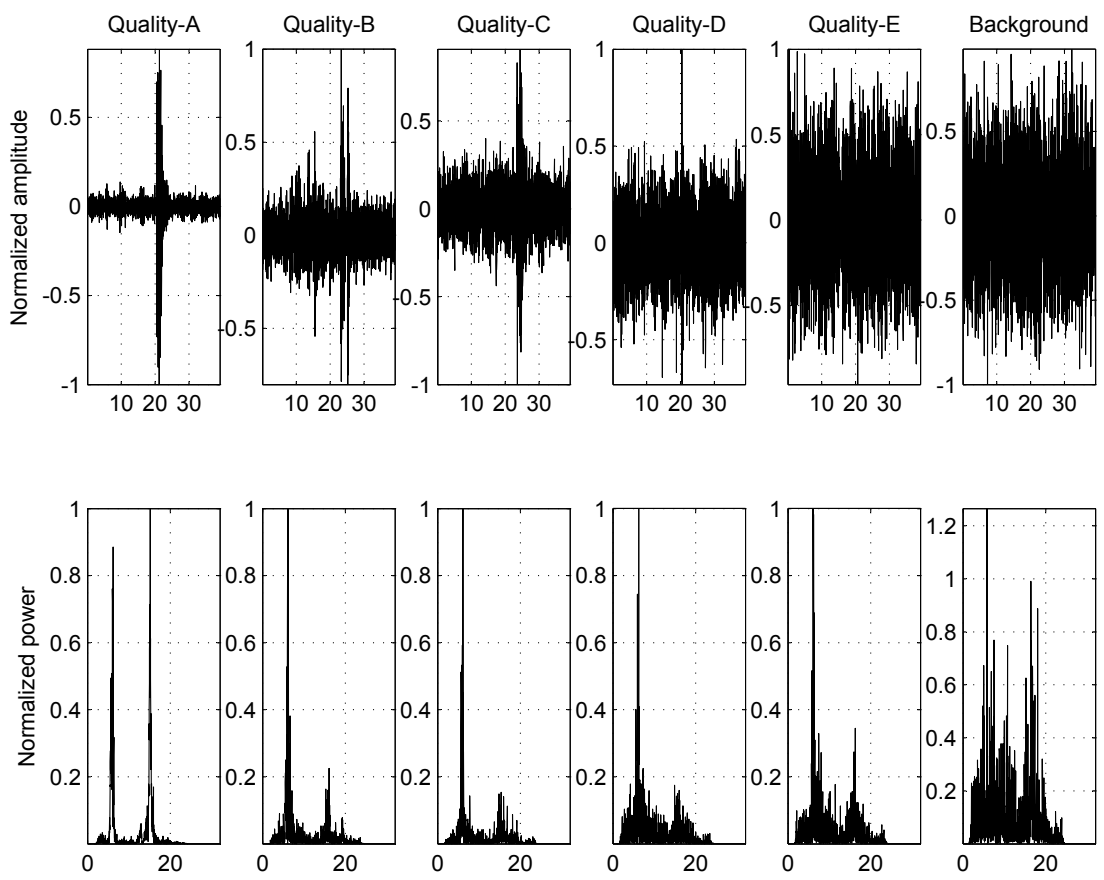

Fig. 1. Average power spectra of six categories of emissions in comparison to the the background. The first row of graphs contains one signal of each quality type. Abscissae of time sequences (ms); abscissae of spectra $(\mathrm{kHz})$. 
Signals have been previously high-pass filtered in order to suppress low frequency components which would mask the higher-frequency components. A 5thorder Butterworth digital filter with cut-off frequency of $2 \mathrm{kHz}$ is used.

Each graph is the result of averaging a number of 15, 2500-sample registers from reticulitermes lucifugus feeding activity. Figure 1 shows the average power spectra of six signal categories according to their amplitude quantization levels. These AE signals were acquired by the sensor SP1-L, using a sampling frequency of $64 \mathrm{kHz}$ and a resolution of 16 bits. These spectra are compared with the background in order to establish an identification criterion. Vibrations of qualities $D$ and $E$ are inaudible.

Quality-A (Q-A) signals' amplitudes belong to the quantization levels interval [25000,30000]. Q-B levels are in [15000, 20000]. Q-C levels are in [10000, 15000]. Quality-D levels are in the interval $[5000,10000]$. Finally, Q-E impulses are completely buried in the background. We are sure about the infestation, so the AE events in this series are due to termite activity, probably in a 3 meter-radius subset within the area under study.

The two main frequency components in the spectra of Figure 1 appear at 6 and $15 \mathrm{kHz}$, respectively, which are associated with the frequency response of the sensor, to the features of the sounds produced by the termite species and to the characteristics of the substratum, through which the emissions propagate. We conclude that using the probe SP1-L we can detect an infestation by interpreting the power spectra diagrams. This is due to the differences that the emissions exhibit in comparison with the flatter shape of the background spectra (sixth column of graphs in Figure 1).

The calculation of 3th-order spectra is performed with a twofold purpose. The first objective is to enhance the detection criteria in the frequency domain. The second purpose is to use more economic sensors, with a lower sensitivity and a higher band-width. Figure 2 shows the average diagonal bi-spectra associated with the signals acquired with the probe SP1-L. A maximum lag $\chi=512$ was selected to compute the third-order auto-cumulants of the signals. The bottom bi-spectrum characterizes the background sound (the most unfavorable, with an amplitude lower in four orders of magnitude than Q-A).

The main frequency component in Figure $2(6 \mathrm{kHz})$ permits to establish a detection criterion based on the identification of this maximum value. It is remarkable that this frequency is also associated with the sensor. Another sensor would display another bi-spectrum shape. For this reason the proposed method of insect detection is based on the prior characterization of the transducer. On the other hand, the magnitudes of the bi-spectra in Figure 2 don't suffer a dramatic attenuation from high to low levels (two orders from Q-A to Q-E). This fact reinforces the criterion of identification, in the sense that it is the noise (symmetrically distributed) which is mainly reduced in the higher-order computation.

On the basis of these results we establish the conclusions related to the identification criterion proposed. 


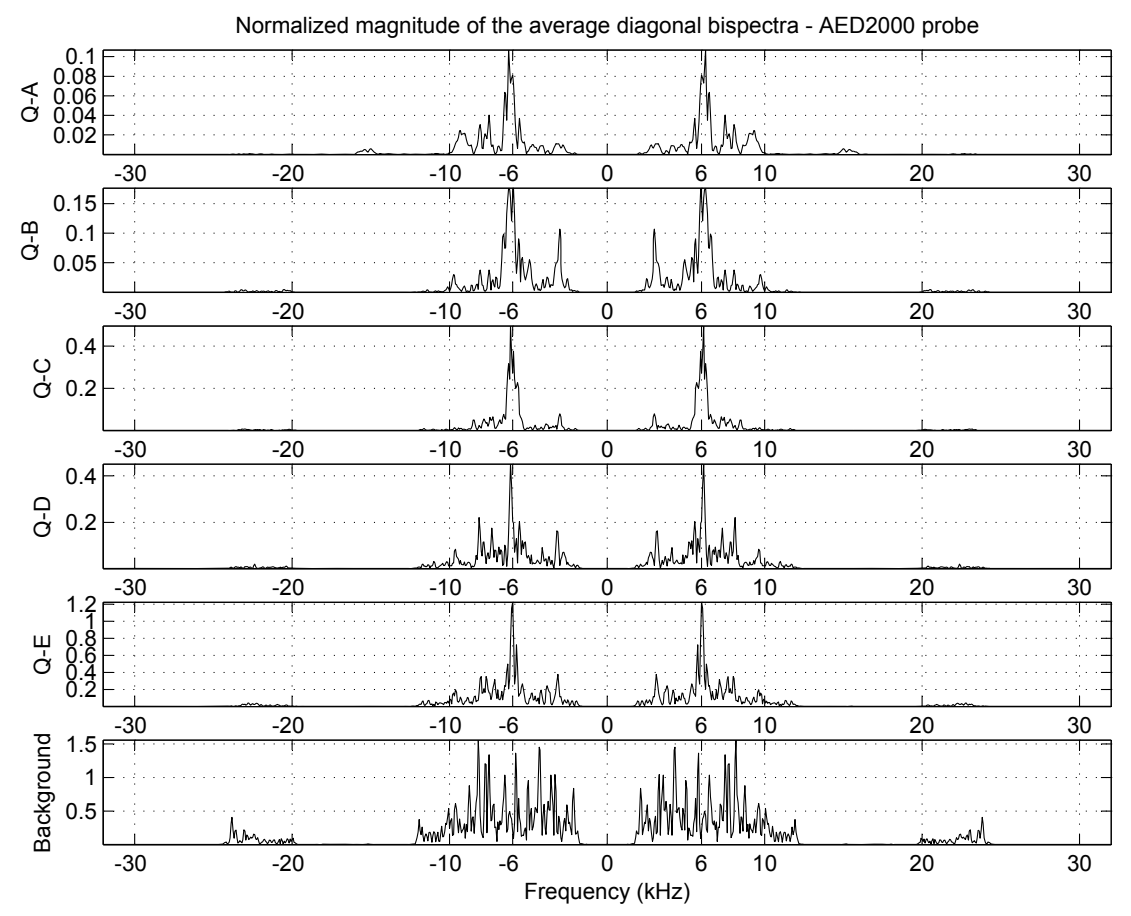

Fig. 2. Average diagonal bi-spectra of signals acquired with the probe SP1-L from AED2000 in comparison to the background sounds, for a maximum lag, $\chi=512$. Each bi-spectrum results from averaging a number of 15, 2500-data registers.

\section{Conclusions}

In this work it has been shown that the diagonal slices of the bi-spectrum are valid and convenient tools for obtaining decision criteria to distinguish a possible infestation, based on the feeding activities of the termites. We have funded this conclusion on three arguments:

First, higher-order cumulants and spectra, as defined herein, enable the signal analysis procedure to have access to waveform information that is typically unavailable when using prior art (second-order) methods. In particular, we remark the enhancement of the frequency diagrams. This is due to the rejection exerted on symmetrically distributed noise processes. In fact, non-Gaussian processes are completely characterized by means of HOS.

Secondly, the potentially valuable information contained in an AE signal (most part of its spectrum) is related to the impulses. The average spectrum reveals amplitude information (the resonance peaks) but phase information is not shown. Higher-order spectra are arrangements of complex numbers and contain this additional information which can be valuable in a pattern recognition or identification criterion context. 
Finally, using different sensors the criterion changes the frequency set-point. Besides, the probability of a false alarm is very low, considering the fact that we had to provide, intentionally, the worst case of background noise. Repeatability has been estimated in a 75 per cent.

Future work is focussed on reducing the computational complexity of HOS in two directions. On one side, we are using compact functions, like FFT and FFTshift. Secondly, we have to adopt a compromise between the maximum lag $(\chi)$ and the resolution, in order to save storage memory and time. These actions are oriented to implement the algorithms in a digital signal processor, in an autonomous hand-instrument.

\section{Acknowledgement}

The authors would like to thank the Spanish Ministry of Education and Science for funding the projects DPI2003-00878, TEC2004-06096 and PTR1995-0824-OP.

\section{References}

1. Röhrig, A., Kirchner, W.H., Leuthold, R.H.: Vibrational alarm communication in the african fungus-growing termite genus macrotermes (isoptera, termitidae). Insectes Sociaux 46 (1999) 71-77

2. De la Rosa, J.J.G., Lloret, I., Puntonet, C.G., Górriz, J.M.: Wavelets transforms applied to termite detection. In: IEEE Third International Workshop on Intelligent Data Acquisition and Advanced Computing Systems: Technology and Applications (IDAACS'2005). Proceedings, Budapest-Hungary, Institute of Computer Information Technologies (2005) oral presentation.

3. Mankin, R.W., Fisher, J.R.: Current and potential uses of acoustic systems for detection of soil insects infestations. In: Proceedings of the Fourth Symposium on Agroacoustic. (2002) 152-158

4. De la Rosa, J.J.G., Puntonet, C.G., Lloret, I., Górriz, J.M.: Wavelets and wavelet packets applied to termite detection. Lecture Notes in Computer Science (LNCS) 3514 (2005) 900-907 Computational Science - ICCS 2005: 5th International Conference, GA Atlanta, USA, May 22-25, 2005, Proceedings, Part I.

5. De la Rosa, J.J.G., Puntonet, C.G., Lloret, I.: An application of the independent component analysis to monitor acoustic emission signals generated by termite activity in wood. Measurement (Ed. Elsevier) 37 (2005) 63-76 Available online 12 October 2004.

6. De la Rosa, J.J.G., Lloret, I., Puntonet, C.G., Górriz, J.M.: Higher-order statistics to detect and characterise termite emissions. Electronics Letters 40 (2004) 1316-1317 Ultrasonics.

7. Nykias, C.L., Mendel, J.M.: Signal processing with higher-order spectra. IEEE Signal Processing Magazine (1993) 10-37

8. Mendel, J.M.: Tutorial on higher-order statistics (spectra) in signal processing and system theory: Theoretical results and some applications. Proceedings of the IEEE 79 (1991) 278-305 